\title{
Qualitative attentional changes with age in doing two tasks at once
}

\author{
François Maquestiaux ${ }^{1}$
}

Published online: 24 June 2015

(C) Psychonomic Society, Inc. 2015

\begin{abstract}
Does practice reduce, or even eliminate, aging effects on the attentional limitations responsible for dual-task interference? The studies reviewed in this article show that age differences reliably persist after extensive practice. Strikingly, dual-task interference remains larger among older adults even in training conditions that allow them to achieve singletask performance as fast as younger adults. These findings demonstrate that age deficits in attentional functioning are robust. Advancing age also can be accompanied by improvements in cognitive functioning, such as in the ability to access the lexicon without attention (i.e., automatically), due to lifelong experience with word reading. Future research needs to establish whether age deficits in central attention are due to structural changes that are irreversible or reversible to some extent.
\end{abstract}

Keywords Dual-task interference $\cdot$ Attention · Aging · Practice $\cdot$ Automatization

Observing the street life from a sidewalk café, I frequently notice young individuals, but rarely elderly adults, doing two tasks at once, such as reading while walking or writing a text message while riding a bike. Why then do older adults apparently avoid placing themselves in such situations? Are they unable to multitask or do they simply choose not to? And, assuming that difficulties with performing two tasks at once (dual-task interference) progressively worsens as people age

François Maquestiaux

francois.maquestiaux@univ-fcomte.fr

1 Université Bourgogne Franche-Comté - UFR SLHS, 30 rue Mégevand, 25030 Besançon, France
(Hartley, 1992), can practice level the playing field? The present review will address these questions.

\section{Cognitive slowing and age deficits}

In many different cognitive domains (e.g., perception, memory, attention, reasoning), a substantial proportion of age differences can be explained by cognitive slowing (Cerella, 1985; Salthouse, 1996). As a rule-ofthumb, older adults' reaction times are approximately 1.5 times slower than younger adults' reaction times. In the domain of dual-task performance, Verhaegen, Steitz, Sliwinski, and Cerella (2003) conducted a careful analysis of the reaction times from 33 studies published between 1962 and 1999. By combining a large number of dual-task studies employing a wide variety of taskse.g., a color-identification task with a letteridentification task (Hartley \& Little, 1999), a visual discrimination task with an auditory search task (McDowd \& Craik, 1988), or a monitoring task with an alphabetic-arithmetic task (Kramer, Larish, \& Strayer, 1995) - Verhaeghen et al. wished to identify the main trend caused by aging on dual-task processing, specifically whether aging provokes some general slowing, some more localized declines, or a combination of both. This meta-analysis confirmed the important role played by generalized slowing and, in addition, identified a general trend for "a significant age-related deficit in dual-task performance" (Verhaegen et al., 2003, p. 451). As I will show next, identifying the exact nature of specific age deficits in dual-task processing benefits from the use of the Psychological Refractory Period (PRP) procedure, which became during the 20th century 
a valuable "microscope" into the nature of attentional mechanisms (Maquestiaux, 2012).

\section{Attentional limitations}

Progress in the understanding of attentional limitations in dual-task situations was spurred by methodological advances, such as precise monitoring of the time course of mental events. These advances made it possible to derive precise predictions from competing models. Of particular note is the PRP procedure, which permits examination of how engaging participants' attention with one task (Task 1) impacts the performance of another task that follows closely thereafter (Task 2). The tasks typically use distinct stimuli, S1 and S2 (e.g., a letter, a tone), requiring distinct responses, R1 and R2 (e.g., pressing a key, saying a word). Researchers sometimes try to minimize peripheral conflicts (e.g., input conflicts or output conflicts) to investigate more directly the nature of "central" attentional limitations. RTs are measured for each task (RT1 and RT2). Critically, there is a variable time between the stimulus onsets - known as the stimulus onset asynchrony (SOA). The temporal overlap between Task-1 processing and Task-2 processing is high with short SOAs (e.g., $15 \mathrm{~ms}$ ) but small with long SOAs (e.g., 1,000 ms). The participant's attention is first engaged on Task 1, because S1 is usually presented before $\mathrm{S} 2$ and because instructions often emphasize rapid Task-1 responses (although not necessarily requiring participants to emit the Task-1 response first). The result is that interference is observed almost exclusively on Task 2 , which greatly simplifies model testing.

A remarkably pervasive phenomenon is that participants are slower on Task 2 (e.g., by $350+\mathrm{ms}$ ) when the SOA is short rather than long. This large RT2 lengthening - the difference between mean RT2 at the shortest SOA and mean RT2 at the longest SOA - is called the PRP effect.

\section{Two theoretical accounts}

Figure 1 illustrates one prominent and well-supported theoretical account of the PRP phenomenon, called the central bottleneck model (Pashler, 1994). Three successive processing stages intervene between stimulus onset and the associated motor response: perceptual encoding (A), central processing (B), and response processing (C). The core assumption is that some limited attentional resource(s) can handle only one central operation (e.g., response selection, memory retrieval) at a time. Because of this bottleneck, central processing (the gray stages in Fig. 1) operates on one task at a time, thus yielding a delay in Task-2 processing (i.e., the horizontal dashed line in Fig. 1). An additional assumption is that peripheral processing (i.e., stages $\mathrm{A}$ and $\mathrm{C}$ ) on one task can be performed in parallel with any processing stage of the other task. As an analogy, imagine that Task- 1 central processing and Task- 2 central processing are two cars waiting to pay a toll. The Task- 2 car must wait for the Task-1 car to complete its transaction. Following this structural account, no processes are actually slowed, but they sometimes must wait for access to a resource.

An alternative theoretical account of the PRP phenomenon assumes that the delay in Task-2 processing is under strategic control (Meyer \& Kieras, 1997a, b). The waiting reflects the voluntary adoption of a task-coordination strategy that delays Task- 2 central processing while Task-1 central processing is underway. Two features of the PRP procedures - namely, stimulus presentation order (S1 then S2) and instructions to emit rapid Task-1 responses - may encourage such a cautious strategy. In this strategic account, parallel central processing would be possible if participants adopted a daring taskcoordination strategy.

According to the central bottleneck model, the size of the PRP effect is greatly influenced by the duration of the perceptual and central stages of Task 1 (i.e., stages 1A and 1B). To understand why, it is useful to express the PRP effect in terms of the durations of Task-1 and Task-2 component processes (for more details, see Pashler \& Johnston, 1989, or Ruthruff, Johnston, \& Van Selst, 2001):

$\mathrm{PRP}$ effect $=1 \mathrm{~A}+1 \mathrm{~B}-2 \mathrm{~A}-\mathrm{SOA}_{\text {short }}$

According to Eq. 1, any manipulation that decreases the length of Task-1 perceptual encoding and/or Task-1 central processing should reduce the size of the PRP effect.

Because RT1 $=1 \mathrm{~A}+1 \mathrm{~B}+1 \mathrm{C}$, it follows that $1 \mathrm{~A}+1 \mathrm{~B}=$ RT1 $-1 \mathrm{C}$. Replacing $1 \mathrm{~A}+1 \mathrm{~B}$ by RT1 $-1 \mathrm{C}$ in Eq. 1 , the PRP equation can be expressed as follow:

PRP effect $=\mathrm{RT} 1-1 \mathrm{C}-2 \mathrm{~A}-\mathrm{SOA}_{\text {short }}$

According to Eq. 2, the size of the PRP effect is influenced by the duration of RT1. Based on this equation, one can predict that the shorter RT1 is, the shorter the PRP effect will be, millisecond for millisecond. Consistent with this prediction, a close correspondence between decreases in RT1 and in the PRP effect has been found in PRP studies (Ruthruff, Johnston, \& Van Selst, 2001; Van Selst, Ruthruff, \& Johnston, 1999).

The prediction that the PRP effect primarily depends upon the length of RT1 is of particular interest for the identification of aging deficits in dual-task interference. According to a quantitative account of age differences, PRP effects should be smaller for older adults relative to younger adults, provided that they are equally fast on Task 1 (i.e., same RT1). The reason is that, due to some generalized slowing, the value of the parameters $-1 \mathrm{C}-2 \mathrm{~A}$ in Eq. 2 should be more negative for older adults than for younger adults, thus yielding a smaller PRP effect in older adults than in younger adults for a given 

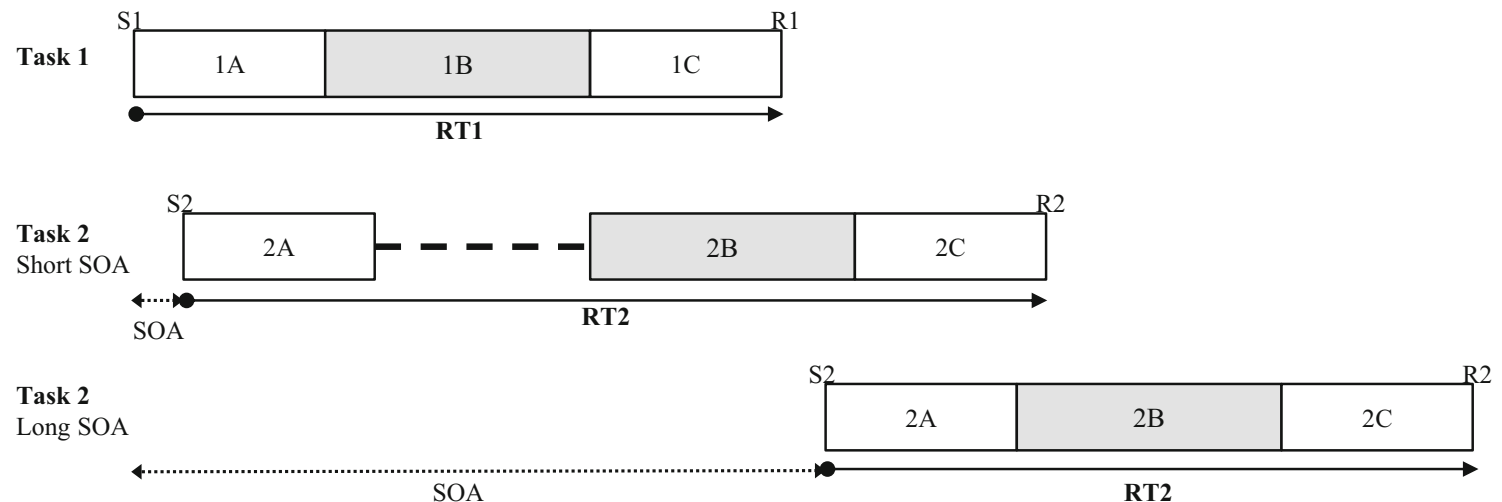

Fig. 1 Central bottleneck model. Central processing proceeds on one task at a time, resulting in a delay on Task 2 (the horizontal dashed line) at the short stimulus onset asynchrony (SOA) but not at the long SOA. A = perceptual encoding; $\mathrm{B}=$ central processing; $\mathrm{C}=$ response processing

RT1. But according to a qualitative account of age differences, PRP effects should be larger for older adults relative to younger adults for a given RT1, provided that one assumption is met. This assumption is that aging profoundly modifies the nature of the central attentional limitation. This modification can, for example, be implemented by incorporating an additional parameter in Eq. 2, only for older adults, called the $S$ parameter. For older adults, the PRP equation can then be rewritten as follows:

PRP effect $=\mathrm{RT} 1-1 \mathrm{C}-2 \mathrm{~A}+\mathrm{S}-\mathrm{SOA}_{\text {short }}$

This additional parameter $S$ may represent an extra time, only needed by older adults, to switch attention from Task-1 central processing to Task-2 central processing at short SOAs (when attention is demanded by the two tasks at the same time) but not at the longest SOA (when attention is demanded by one task at a time).

To summarize, whereas a quantitative model of age differences predicts smaller PRP effects for older adults than for younger adults even if RT1 is equal between the two age groups, the qualitative model of age differences predicts larger PRP effects for older relative to younger adults for a given RT1 (because of the presence of an additional parameter $S$ in the PRP equation, only for older adults). These opposing predictions about the nature of dual-task deficits with aging (quantitative change vs. qualitative change) are evaluated in the experiments reviewed below.

\section{Quantitative or qualitative change with age?}

It is well-documented that PRP interference generally increases with advancing age. For instance, Allen, Smith, Vires-Collins, and Sperry (1998) reported a $210 \%$ increase in PRP effects in older adults (322 ms) relative to younger adults (104 ms). Empirical studies have proposed three sources of
PRP increases with aging: a specific lengthening of the duration of central processing (Allen et al., 1998), a general lengthening of all processing stages (Hartley \& Little, 1999), and the adoption of conservative task-coordination strategies by older adults (Glass et al., 2000).

Another approach to understand age effects on attentional functioning is to examine whether quantitative age differences in PRP interference can be reduced or even eliminated with practice. Using this approach, Maquestiaux, Hartley, and Bertsch (2004) asked six younger adults $(M=23.5$ years) and six older adults $(M=65.2$ years $)$ to perform 2,240 dualtask trials spread over seven experimental sessions. On each trial, participants performed an auditory-vocal Task 1 (identifying a tone among four possible tones) and a visual-manual Task 2 (identifying a letter or a digit among eight possible characters), using the PRP procedure. Because the two tasks have distinct sensory inputs and outputs, this minimized peripheral interference known to be inflated with aging at the level of inputs (Hartley, 2001; Hein \& Schubert, 2004) and motor outputs (Hartley, 2001; Hartley \& Maquestiaux, 2007). Therefore, this procedure is ideal to selectively assess aging effects on central attentional limitations. Practice reduced the overall size of the PRP effect, from $498 \mathrm{~ms}$ in the first session to $267 \mathrm{~ms}$ in the final session. Despite training, older adults still showed larger PRP effects than young adults. In fact, training actually amplified PRP differences between younger and older adults, from $205 \mathrm{~ms}$ early in practice (395 vs. 600 $\mathrm{ms})$ to $282 \mathrm{~ms}$ late in practice (126 vs. $408 \mathrm{~ms}$ ). This amplification resulted from a smaller reduction of PRP interference in older adults relative to younger adults (32\% vs. $69 \%)$. In other words, the age effect on dual-task interference was compounded by an age effect on learning.

Figure 2 (top panel) shows, for each age group, the mean PRP effect against mean RT1 for each of the seven dual-task sessions in Maquestiaux et al. (2004). Consistent with the hypothesis of a specific dual-task deficit due to a qualitative change with age, older 


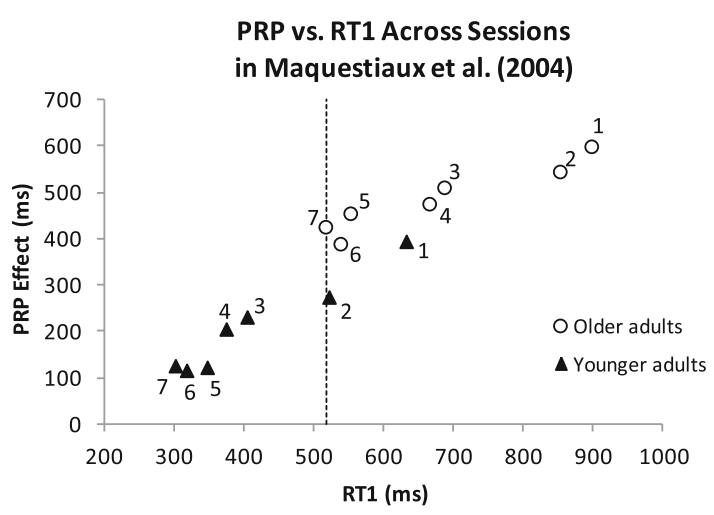

PRP vs. RT1 Across Sessions

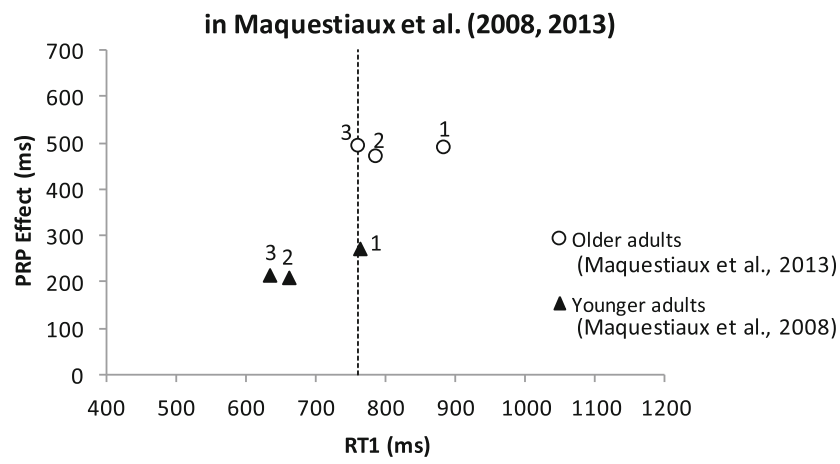

Fig. 2 Mean psychological refractory period (PRP) effect plotted against mean Task-1 reaction time (RT1) for both age groups in different studies. Each point corresponds to one of the seven dual-task sessions in Maquestiaux et al. (2004) [top panel] or one of the three dual-task sessions in Maquestiaux et al. $(2008,2013)$ [bottom panel]. Open circles represent the older adults and filled triangles represent the younger adults. Vertical dashed lines show that older adults' PRP effect is consistently larger than younger adults' PRP effect for the same RT1

adults' PRP effect in session 7 (426 ms) was 151-ms larger $(p<0.05)$ than younger adults' PRP effect in session 2 (275 ms), despite similar RT1s (approximately $520 \mathrm{~ms}$ for both age groups). Clearly, aging exacerbates central attention limitations (whether structural or strategic), causing increased dual-task interference.

\section{Reduced ability with age to automatize a new task}

It often is assumed that practice provokes qualitative changes in the way a task is performed (Moors \& De Houwer, 2006). One widely held view is that task processing eventually becomes "automatic" with practice, operating without recruiting attention (Shiffrin \& Schneider, 1977). When applying this view of automaticity to aging and dual-task interference, an important question arises: Can older adults eliminate central attentional limitations in dual-task situations through task automatization?

The above question is a continuation of research revealing younger adults' ability to process simultaneously the central operations of two distinct tasks under specific experimental conditions (Ruthruff, Van Selst, Johnston, \& Remington, 2006; Maquestiaux, Laguë-Beauvais, Ruthruff, \& Bherer, 2008). For instance, Maquestiaux et al. (2008) asked younger and older adults to learn to perform rapidly a very simple auditory-vocal task (identifying a tone as high or low in pitch) across six training sessions totaling of 5,040 trials. Then, to probe the automaticity of the highly practiced task, these participants performed three dual-task sessions: The practiced task was presented as Task 2 with an unpracticed visualmanual Task 1 (identifying a letter or a digit among 8 possible characters), using the PRP procedure. Two converging tests indicate whether Task 2 needs attention to operate (nonautomatic processing) or operates without attention (automatic processing) following practice. In the case of non-automatic processing, central processing should be serial (Fig. 3A). As a result, the PRP effect should be large (due to the bottleneck delay) and responses should rarely be reversed (i.e., R1 should come before R2 on almost every trial). However, in the case of task automatization, central processing should be parallel (Fig. 3B). As a result, the PRP effect should be small (because Task-2 processing is not delayed) and responses should be frequently reversed (i.e., R2 before R1) at short SOAs (because the practiced Task 2 is much faster than the unpracticed Task 1).

In Maquestiaux et al. (2008), 17 of 20 younger adults $(M=$ 24.6 years) reached the criteria for parallel central processing: the mean PRP effect was unusually small $(166 \mathrm{~ms})$ given the long duration of Task $1(641 \mathrm{~ms})$, and the percentage of response reversals was high at the shortest SOA (66.1\%). Meanwhile, in a study of older adults using the exact same

(A) Serial Central Processing

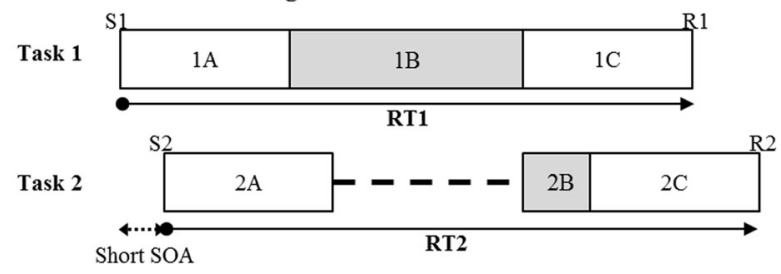

(B) Parallel Central Processing

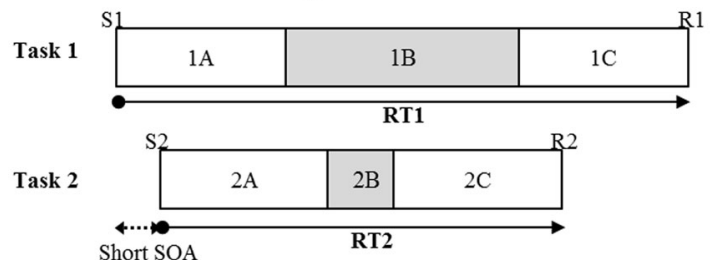

Fig. 3 Processing time diagrams at short stimulus onset asynchrony (SOA) when pairing an unpracticed Task 1 (thus yielding long central processing) with a highly practiced Task 2 (thus yielding short central processing). A In the case of serial central processing, Task-2 processing is delayed and Task-1 response is followed by Task-2 response. B In the case of parallel central processing due to the automatization of Task-2 central processing, Task-2 processing is uninterrupted and response order is reversed. $\mathrm{A}=$ perceptual encoding; $\mathrm{B}=$ central processing; $\mathrm{C}=$ response processing 
experimental conditions (Maquestiaux et al., 2010), only one older adult out of $12(M=63.3$ years) reached the criteria for parallel central processing (i.e., PRP effect of $245 \mathrm{~ms}, 60.2 \%$ of response reversals at the shortest SOA), whereas the vast majority of the older adults (11/12) were unable to do so, as evidenced by their large mean PRP effect (541 ms) and their low percentage of response reversals at the shortest SOA $(8.3 \%)$. In other words, the prevalence of individuals able to reach parallel central processing was 10 times greater among younger adults $(85 \%)$ than among older adults $(8 \%)$.

One interpretation is that aging provokes a qualitative reduction in plasticity - an inability to automatize new taskspreventing the elimination of central attentional limitations with training. Another interpretation is that older adults merely learn more slowly and therefore did not get the chance to reach the performance threshold necessary for entire task automatization (Maquestiaux et al., 2010); after the six training sessions, their mean RT on the auditory-vocal task was still 222 ms longer than that of the younger adults (530 vs. 308 $\mathrm{ms})$.

The two possible interpretations of (most) older adults' failure to reach parallel central processing (i.e., an inability to automatize new tasks or a slower rate of learning) were tested by Maquestiaux et al. (2013). Maquestiaux et al. (2013) tested 10 new older adults ( $M=65.6$ years) using the previous procedure (Maquestiaux et al., 2008, 2010) with two changes. The first change was to double the amount of training on the auditory-vocal task: 10,080 trials spread over 12 sessions, instead of 5,040 trials over 6 sessions. The second change was to simplify both the auditory-vocal and visualmanual tasks (e.g., use of more discernible tone pitches). Although this even more extensive training on even easier tasks reduced older adults' terminal RTs to only $307 \mathrm{~ms}$, a value virtually identical to that attained by younger adults with half the training (Maquestiaux et al., 2008), the majority of older adults (i.e., 8/10) nevertheless still were unable to achieve parallel central processing: The mean PRP effect was large $(538 \mathrm{~ms})$ and responses were rarely reversed at the shortest SOA (4.6\%). This finding is strongly suggestive of a qualitative change in dual-task processing with age.

\section{Age-related deficit or mere cognitive slowing?}

Can mere cognitive slowing explain the change in PRP performance with age? Fig. 2 (bottom panel) shows, for each age group, the mean PRP effect against mean RT1 for each of the three dual-task PRP sessions in our study (Maquestiaux et al., 2013). Again, and consistent with the findings from Maquestiaux et al.'s (2004) mentioned above, the results are incompatible with the prediction of a quantitative account of age deficits in dual-task interference (i.e., smaller PRP effects for older adults relative to younger adults for a same RT1).
First, older adults' PRP effect in session 3 (497 ms) is $224 \mathrm{~ms}$ larger than younger adults' PRP effect in session 1 (273 ms), even though both age groups had a similar RT1 of about 760 ms. Second, older adults' response reversal rate (at the shortest $\mathrm{SOA})$ in session $3(5.6 \%)$ is much smaller than younger adults' response reversal rate in session 1 (47.7\%). The failure of a majority of older adults to eliminate central attentional limitations cannot be attributed merely to a generalized cognitive slowing.

\section{What is the nature of age-related deficits?}

The robust age deficits identified in central processing can be accounted for by a central bottleneck that becomes more rigid with age, in the sense of a lost ability to automatize task performance. Logan (1988) proposed that training shortens RT because of a gradual shift from slow algorithmic-based processing to fast memory-based processing. Following this proposal, younger and older adults may have reached nearly identical RTs in two different ways: a shift toward fast, automatic memory-based processing for younger adults but a mere speed-up of algorithmic-based processing for older adults. This hypothesis could explain why younger adults reached parallel central processing but older adults did not, despite equal processing speed. Note that recent findings in the domain of numerical cognition echo the proposal of two distinct processing modes that can both achieve fast RTs. For instance, when participants performed simple addition and subtraction problems, Fayol and Thevenot (2012), followed by Barrouillet and Thevenot (2013), observed that compacted counting procedures (similar to algorithmic-based processing) can be as fast as direct retrieval of the answer from long-term memory (similar to memory-based processing).

An alternative explanation is that age deficits are strategic in nature, reflecting increased conservatism regarding processing overlap (Glass et al., 2000). This conjecture is not easily tested with the PRP procedure. Ironically, the PRP procedure's main virtue (loading all the interference on one task) is also its main weakness (possibly encouraging a strategy of task postponement). In training experiments encouraging processing overlap, such as by using simultaneous presentation of the stimuli and instruction to treat both tasks equally, very little dual-task interference was found in young adults (Hazeltine, Teague, \& Ivry, 2002; Schumacher et al., 2001; for similar evidence without training but with ideomotor compatible tasks, see Halvorson, Ebner, \& Hazeltine, 2013) but relatively large amounts were observed in older adults (Strobach, Frensch, Müller, \& Schubert, 2012a, b). These findings suggest that strategic factors do not fully account for age deficits in dual-task interference. In other words, I propose that structural changes of the central bottleneck cause a sizable portion of age deficits. 


\section{Evidence for automatic processing in old age}

If older adults' dual-task deficits merely reflect conservative processing strategies, then one might expect them to demonstrate less automaticity in all domains. This is clearly not the case. Given the considerable cumulative exposure that older adults have with word reading relative to younger adults, Lien et al. (2006) wondered whether some specific underlying cognitive operations can proceed automatically. Specifically, Lien et al. tested the hypothesis that aging develops the ability to recognize words while central attention is devoted to another task. To test this hypothesis, younger and older adults performed a discrimination Task 1 that was either visual (identify the shape of a visual stimulus) or auditory (identify the pitch of an auditory stimulus), along with a lexical-decision Task 2 (determine whether a string of letters forms a word or a nonword), using the PRP procedure. The authors manipulated the frequency of the words used on Task 2, with high-frequency words expected to produce shorter RTs relative to lowfrequency words, thus creating a word frequency effect.

Lien et al. (2006) derived two distinct sets of predictions, according to whether word recognition needs attention or can operate automatically. If Task-2 lexical processing cannot operate while central attention is devoted to Task-1 processing, then the word frequency effect on RT2 should be additive with SOA (same effect size at every SOA). But if Task-2 lexical processing operates automatically, then word frequency should interact underadditively with SOA; that is, the frequency effect on RT2 should be much smaller (or even null) at the shortest SOA (when the central bottleneck delays Task-2 processing) relative to the longer SOAs, due to some "absorption" of word-frequency effects into the central bottleneck delay (i.e., the horizontal dashed line in Fig. 1). The effect of word frequency with SOA on RT2 was approximately additive for younger adults but strongly underadditive for older adults (whether Task-1 stimulus was visual or auditory), a pattern of findings consistent with non-automatic processing for younger adults but with automatic processing for older adults. This greater automaticity with advancing age (for converging evidence, see, e.g., Allen, Lien, Murphy, Sanders, Judge, \& McCann, 2002) likely reflects older adults' lifelong exposure with reading (for evidence that greater lexical skill leads to greater automaticity, see Ruthruff, Allen, Lien, \& Grabbe, 2008).

It is worth pointing out that Lien et al.'s (2006) discovery of an improved efficiency of lexical access with age was accompanied by larger PRP effects for older adults than for younger adults (511 ms vs. $321 \mathrm{~ms})$. Such coexistence of losses and gains in older adults' cognitive functioning is indicative of their ability to rely on "pockets" of cognitive strength (i.e., automatic lexical access), even though they experience qualitative age deficits in central attention (i.e., response selection). But adults' more automatic lexical processing was insufficient to compensate fully for their deficits at the level of central processes, thus explaining why they still experienced larger PRP effects.

Besides word recognition, processes involved in emotional expression (an activity on which individuals have a lot of expertise due to considerable exposure in everyday life) can evade the central bottleneck (i.e., thus proceeding automatically). For instance, in a PRP experiment using event-related potential (ERP) measures, Pollock et al. (2012) found that younger and older adults were able to perceive the emotion conveyed by a face (presented as a Task- 2 stimulus) while central attention was devoted to the processing of a tone discrimination task (Task 1). Specifically, the P1 component - an electrophysiological measure sensitive to emotional processing - had much higher amplitudes at short than at long SOAs for both age groups. This study is suggestive of preserved automatic processing of emotion conveyed by Task 2 faces in old age.

Using highly compatible stimuli and responses as Task 2 in PRP experiments, Hartley, Seaman, and Maquestiaux (2015) found that some central processes associated with a saccade or a body tilt (i.e., a highly-practiced response throughout the life) in the direction of rotation of a visual stimulus could operate automatically while central attention was devoted to another task and that this ability was preserved in old age. The list of cognitive processes known to operate automatically, even in old age (i.e., word reading, saccade, body tilt), lengthens but is probably limited to those skills that gradually develop over the life span. To date, there is no evidence that typical older adults can learn to perform a new skill, on which they have not had considerable prior experience, without central attention.

\section{Conclusions}

A sizable amount of the age differences in dual-task performance stems from robust age-related deficits in the central attentional limitation that prevents two "thoughtlike" processes (e.g., those involved in deciding how to deal with the current stimulus) to operate at the same: In conditions minimizing peripheral conflicts (between inputs and between outputs), dual-task interference is still much more pronounced in older adults. This is true despite several thousands of training trials and despite achieving single-task RTs equal to those of younger adults (Maquestiaux et al., 2013). Because eliminating dual-task interference between novel tasks seems nearly impossible for older adults, I argue for a profound qualitative change with age in the way attention is allocated between tasks.

Strikingly, the qualitative deficit with age in dual-task performance may not stem from a problem with automaticity per se, because older adults can retain automaticity developed 
across lifespan. For instance, Lien et al. (2006) provided empirical evidence consistent with greater automatization in lexical access for older adults relative to younger adults, due to lifelong cumulative exposure to word reading. Therefore, the reduced ability to automatize task performance appears specific to new tasks, certainly because the amount of practice on a new task that one can accumulate will remain much smaller when starting practice at the age of 60 years rather than at the age of 20 years. Such difficulty of acquiring new automaticity in old age might help to explain the observation that, unlike younger adults, older adults rarely seem to perform two tasks at once in daily life.

The discovery of severe deficits with age in central attention is consistent with a decreased plasticity of attentional systems. Is this modification totally irreversible or, in contrast, is there some room for some improvement (i.e., for some increased plasticity)? Future research addressing this question may be inspired by findings from the field of associative learning. For instance, there is some evidence that strong incentives (e.g., financial rewards indexed on speed) render older adults more likely to shift from a time-consuming algorithmic strategy to a more automatic memory-retrieval strategy when performing a computation-based task (Touron \& Hertzog, 2009).

The conclusion of qualitative changes with age in central attention raises intriguing research questions in other domains, such as walking or working memory. Are these attentional changes responsible for older adults' reduced ability to perform concurrently a novel cognitive task while walking (Siu et al., 2008)? Because processing and maintenance of information in working memory may be constrained by a processing bottleneck (Barrouillet, Bernardin, \& Camos, 2004), are the qualitative changes with age in central attention responsible for aging effects on working memory (Bopp \& Verhaeghen, 2007)? Questions such as these deserve consideration given the importance of walking in independent living and the crucial role played by working memory in complex skills, such as typewriting (Yamaguchi \& Logan, 2014), and in numerous higher-order cognitive tasks, such as reading, writing, and reasoning (Engle, 2002).

Acknowledgments This work was supported by PIR and PICS grants from Centre National de la Recherche Scientifique.

\section{References}

Allen, P. A., Smith, A. F., Vires-Collins, H., \& Sperry, S. (1998). The psychological refractory period: Evidence for attentional differences in time-sharing. Psychology and Aging, 13, 218-229.

Allen, P. A., Lien, M.-C., Murphy, M. D., Sanders, R. E., Judge, K. S., \& McCann, S. R. (2002). Age differences in overlapping-task performance: Evidence for efficient parallel processing in older adults. Psychology and Aging, 17, 505-519.
Barrouillet, P., \& Thevenot, C. (2013). On the problem-size effect in small additions: Can we really discard any counting-based account? Cognition, 128, 35-44.

Barrouillet, P., Bernardin, S., \& Camos, V. (2004). Time constraints and resource sharing in adults' working memory spans. Journal of Experimental Psychology: General, 133, 83-100.

Bopp, K. L., \& Verhaeghen, P. (2007). Age-related differences in control processes in verbal and visuospatial working memory: Storage, transformation, supervision, and coordination. Journal of Gerontology: Psychological Sciences, 62, 239-246.

Cerella, J. (1985). Information processing rates in the elderly. Psychological Bulletin, 98, 67-83.

Engle, R. W. (2002). Working memory capacity as executive attention. Current Directions in Psychological Science, 11, 19-23.

Fayol, M., \& Thevenot, C. (2012). The use of procedural knowledge in simple addition and subtraction problems. Cognition, 123, 392-403.

Glass, J. M., Schumacher, E. H., Lauber, E. J., Zurbriggen, E. L., Gmeindl, L., Kieras, D. E., \& Meyer, D. E. (2000). Aging and the psychological refractory period: Task-coordination strategies in young and old adults. Psychology and Aging, 15, 571-595.

Halvorson, K. M., Ebner, H., \& Hazeltine, E. (2013). Investigating perfect timesharing: The relationship between IM-compatible tasks and dual-task performance. Journal of Experimental Psychology: Human Perception and Performance, 39, 413-432.

Hartley, A. A. (1992). Attention. In F. I. M. Craik \& T. A. Salthouse (Eds.), The handbook of aging and cognition (pp. 3-49). Hillsdale, NJ: Erlbaum.

Hartley, A. A. (2001). Age differences in dual-task interference are localized to response generation processes. Psychology and Aging, 16, $47-54$.

Hartley, A. A., \& Little, D. M. (1999). Age-related differences and similarities in dual-task interference. Journal of Experimental Psychology: General, 128, 417-450.

Hartley, A. A., \& Maquestiaux, F. (2007). Success and failure at dual-task coordination by younger and older adults. Psychology and Aging, 22, 215-222.

Hartley, A. A., Seaman, B., \& Maquestiaux, F. (2015). Ideomotorcompatible tasks partially escape dual-task interference in both young and elderly adults. Psychology and Aging, 30, 36-45.

Hazeltine, E., Teague, D., \& Ivry, B. (2002). Simultaneous dual-task performance reveals parallel response selection after practice. Journal of Experimental Psychology: Human Perception and Performance, 28, 527-545.

Hein, G., \& Schubert, T. (2004). Aging and input processing in dual-task situations. Psychology and Aging, 19, 416-432.

Kramer, A. F., Larish, J., \& Strayer, D. L. (1995). Training for attentional control in dual-task settings: A comparison of young and old adults. Journal of Experimental Psychology: Applied, 1, 50-76.

Lien, M.-C., Allen, P. A., Ruthruff, E., Grabbe, J., McCann, R. S., \& Remington, R. W. (2006). Visual word recognition without central attention: Evidence for greater automaticity with advancing age. Psychology and Aging, 21, 431-447.

Logan, G. D. (1988). Toward an instance theory of automatization. Psychological Review, 95, 492-527.

Maquestiaux, F. (2012). The simultaneity of mental acts: Contributions from the PRP procedure. L'Année Psychologique/Topics in Cognitive Psychology, 112, 631-663.

Maquestiaux, F., Hartley, A. A., \& Bertsch, J. (2004). Can practice overcome age-related differences in the psychological refractory period effect? Psychology and Aging, 19, 649-667.

Maquestiaux, F., Laguë-Beauvais, M., Ruthruff, E., \& Bherer, L. (2008). Bypassing the central bottleneck after single-task practice in the psychological refractory period paradigm: Evidence for task automatization and greedy resource recruitment. Memory \& Cognition, 36, 1262-1282. 
Maquestiaux, F., Laguë-Beauvais, M., Ruthruff, E., Hartley, A. A., \& Bherer, L. (2010). Learning to bypass the central bottleneck: Declining automaticity with advancing age. Psychology and Aging, 25, 177-192.

Maquestiaux, F., Didierjean, A., Ruthruff, E., Chauvel, G., \& Hartley, A. A. (2013). Lost ability to automatize task performance in old age. Psychonomic Bulletin \& Review, 20, 1206-1212.

McDowd, J. M., \& Craik, F. I. (1988). Effects of aging and task difficulty on divided attention performance. Journal of Experimental Psychology: Human Perception and Performance, 14, 267-280.

Meyer, D. E., \& Kieras, D. E. (1997a). A computational theory of executive cognitive processes and multiple-task performance: Part 1. Basic mechanisms. Psychological Review, 104, 3-65.

Meyer, D. E., \& Kieras, D. E. (1997b). A computational theory of executive cognitive processes and multiple-task performance: Part 2. Accounts of psychological refractory-period phenomena. Psychological Review, 104, 749-791.

Moors, A., \& De Houwer, J. (2006). Automaticity: A theoretical and conceptual analysis. Psychological Bulletin, 132, 297-326.

Pashler, H. (1994). Dual-task performance in simple tasks: Data and theory. Psychological Bulletin, 116, 220-244.

Pashler, H., \& Johnston, J. C. (1989). Chronometric evidence for central postponement in temporally overlapping tasks. Quarterly Journal of Experimental Psychology, 41A, 19-45.

Pollock, J. W., Khoja, N., Kaut, K. P., Lien, M.-C., \& Allen, P. A. (2012). Electrophysiological evidence for adult age-related sparing and decrements in emotion perception and attention. Frontiers in Integrative Neuroscience, 6, 1-14.

Ruthruff, E., Johnston, J. C., \& Van Selst, M. (2001). Why practice reduces dual-task interference. Journal of Experimental Psychology: Human Perception and Performance, 27, 3-21.

Ruthruff, E., Van Selst, M., Johnston, J. C., \& Remington, R. W. (2006). How does practice reduce dual-task interference: Integration, automatization, or just stage-shortening? Psychological Research, 70, $125-142$.

Ruthruff, E., Allen, P. A., Lien, M.-C., \& Grabbe, J. (2008). Visual word recognition without central attention: Evidence for greater automaticity with greater reading ability. Psychonomic Bulletin \& Review, 15, 337-343.

Salthouse, T. A. (1996). The processing-speed theory of adult age differences in cognition. Psychological Review, 103, 403-428.

Schumacher, E. H., Seymour, T. L., Glass, J. M., Fencsik, D. E., Lauber, E. J., Kieras, D. E., \& Meyer, D. E. (2001). Virtually perfect time sharing in dual-task performance: Uncorking the central cognitive bottleneck. Psychological Science, 12, 101-108.

Shiffrin, R. M., \& Schneider, W. (1977). Controlled and automatic human information processing: II. Perceptual learning, automatic attending, and a general theory. Psychological Review, 84, 127-190.

Siu, K. C., Chou, L. S., Mayr, U., van Donkelaar, P., \& Woollacott, M. H. (2008). Does inability to allocate attention contribute to balance constraints during gait in older adults? Journal of Gerontology: Series A: Biological Sciences and Medical Sciences, 63A, 1364 1369.

Strobach, T., Frensch, P., Müller, H., \& Schubert, T. (2012a). Age- and practice-related influences on dual-task costs and compensation mechanisms under optimal conditions of dual-task performance. Aging, Neuropsychology, and Cognition, 19, 222-247.

Strobach, T., Frensch, P. A., Müller, H., \& Schubert, T. (2012b). Testing the limits of optimizing dual-task performance in younger and older adults. Frontiers in Human Neuroscience, 6, 39.

Touron, D. R., \& Hertzog, C. (2009). Age differences in strategic behavior during a computation-based skill acquisition task. Psychology and Aging, 24, 574-585.

Van Selst, M., Ruthruff, E., \& Johnston, J. C. (1999). Can practice eliminate the psychological refractory period effect? Journal of Experimental Psychology: Human Perception and Performance, $25,1268-1283$.

Verhaegen, P., Steitz, D. W., Sliwinski, M. J., \& Cerella, J. (2003). Aging and dual-task performance: A meta-analysis. Psychology and Aging, 18, 443-460.

Yamaguchi, M., \& Logan, G. D. (2014). Pushing typists back on the learning curve: Revealing chunking in skilled typewriting. Journal of Experimental Psychology: Human Perception and Performance, 40, 592-612. 